\title{
Comparative Effects of Herbicide Dicamba and Related Compound on Plant Mitochondrial Bioenergetics
}

\author{
Francisco Peixoto, ${ }^{1}$ Joaquim A. F. Vicente, ${ }^{2}$ and Vitor M. C. Madeira ${ }^{3}$ \\ ${ }^{1}$ Departamento de Química, Universidade de Trás-os-Montes e Alto Douro, 5001 Vila Real, Portugal; E-mail: fpeixoto@utad.pt \\ ${ }^{2}$ Departamento de Botânica, Universidade de Coimbra, 3049 Coimbra, Portugal \\ ${ }^{3}$ Departamento de Bioquímica, Universidade de Coimbra, 3049 Coimbra, Portugal
}

Received 14 February 2003; revised 9 April 2003; accepted 8 May 2003

\begin{abstract}
The herbicide dicamba (3,6-dichloro-2methoxybenzoic acid) was evaluated for its effects on bioenergetic activities of potato tuber mitochondria to elucidate putative mechanisms of action and to compare its toxicity with 2-chlorobenzoic acid. Dicamba (4 $\mu \mathrm{mol} / \mathrm{mg}$ mitochondrial protein) induces a limited stimulation of state 4 respiration of ca. $10 \%$, and the above concentrations significantly inhibit respiration, whereas 2-chlorobenzoic acid maximally stimulates state 4 respiration (ca. 50\%) at about $25 \mu \mathrm{mol} / \mathrm{mg}$ mitochondrial protein. As opposed to these limited effects on state 4 respiration, transmembrane electrical potential is strongly decreased by dicamba and 2chlorobenzoic acid. Dicamba $(25 \mu \mathrm{mol} / \mathrm{mg}$ mitochondrial protein) collapses, almost completely, $\Delta \psi$; similar concentrations of 2-chlorobenzoic acid promote $\Delta \psi$ drops of about 50\%. Proton permeabilization partially contributes to $\Delta \psi$ collapse since swelling in Kacetate medium is stimulated, with dicamba promoting a stronger stimulation. The $\Delta \psi$ decrease induced by dicamba is not exclusively the result of a stimulation on the proton leak through the mitochondrial inner membrane, since there was no correspondence between the $\boldsymbol{\Delta} \psi$ decrease and the change on the $\mathbf{O}_{2}$ consumption on state 4 respiration; on the contrary, for concentrations above $8 \mu \mathrm{mol} / \mathrm{mg}$ mitochondrial protein a strong inhibition was observed. Both compounds inhibit the activity of respiratory complexes II and III but complex IV is not significantly affected. Complex I seems to be sensitive to these xenobiotics. In conclusion, dicamba is a stronger mitochondrial respiratory chain inhibitor and uncoupler as compared to 2-chlorobenzoic acid. Apparently, the differences in the lipophilicity are related to the different activities on mitochondrial bioenergetics. (C) 2003 Wiley Periodicals, Inc. J Biochem Mol Toxicol 17:185-192, 2003; Published online in Wiley InterScience (www.interscience.wiley.com). DOI 10.1002/jbt.10077
\end{abstract}

Correspondence to: Francisco Peixoto.

Contract Grant Sponsor: University of Trás-os-Montes and Alto Douro.

(c) 2003 Wiley Periodicals, Inc.
KEYWORDS: Herbicide; Mitochondria; Solanum tuberosum; Aromatic Carboxylic Acids; Dicamba

\section{INTRODUCTION}

Studies of the effects of herbicides and other xenobiotics on cellular metabolism are mandatory to understand the mechanisms of action. The mitochondrial fraction is a good model for the study of cell toxicity of xenobiotics in energy metabolism, since data from mitochondrial studies are reasonably correlated with cytotoxicity parameters reported for cell cultures and whole organisms [1-3]. These organelles provide the eukaryotic cell of nonphotosynthetic tissue with more than $90 \%$ of its total energy requirements. Suspensions of plant mitochondria can be obtained with a high degree of purity and integrity [4] and changes in activity can be studied by several methods: ion permeability, swelling, polarography, evaluation of transmembrane potentials and enzyme activities. Several xenobiotics interact with mitochondria, being classified, according to their effects, as uncoupling agents, electrontransport inhibitors, or energy-transfer inhibitors [5].

There are no studies of dicamba (3,6-dichloro-2methoxybenzoic acid) effects on the mitochondria and the few published studies of the effects of dicamba were carried out on cells, plants, and animals and have only an agrochemical purpose [6-9].

Aquatic plant toxicity tests are frequently performed as environmental risk assessments to determine the potential impacts of contaminants on primary producers; tests performed in Slenastrum and Lemna do not show any sensitivity to dicamba [10].

Dicamba has been studied for its bioenergetics toxicity interactions in comparison to 2-chlorobenzoic acid, because of the similarities of their chemical structures (Figure 1). These studies are of obvious relevance to toxicology since most of the energetic and intermediary 
A

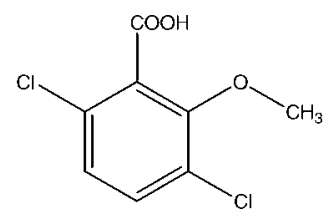

B

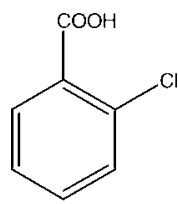

FIGURE 1. Structures of dicamba (A) and 2-chlorobenzoic acid (B).

metabolic compounds are generated at the expense of mitochondrial respiration. Therefore, interactions of xenobiotics with mitochondrial functions may severely affect the general metabolism.

\section{EXPERIMENTAL METHODS}

\section{Preparation of Mitochondria}

Potato tubers (Solanum tuberosum, L.) were obtained from the local market. Mitochondria were isolated and purified according to a procedure involving Percoll gradient centrifugation as a terminal purification step [4], except that $22 \%$ Percoll instead of $28 \%$ was used. The mitochondrial fraction was collected from the Percoll gradient with a Pasteur pipette and washed twice by centrifugation at $30,000 \times g$ for $5 \mathrm{~min}$ in a washing medium containing mannitol $(300 \mathrm{mM})$, bovine serum albumin (BSA; 0.1\%), and Hepes (10 mM; pH 7.2). Protein was determined by the biuret method, using BSA as standard [11].

\section{Measurement of Respiratory Activities}

Oxygen consumption of isolated mitochondria was measured polarographically using a Clark-type oxygen electrode connected to a suitable recorder, in a closed water-jacketed 1-mL chamber with magnetic stirring, at $25^{\circ} \mathrm{C}$ [12]. Respiration rates were calculated assuming an oxygen concentration of $500 \mathrm{nAt} \mathrm{O} / \mathrm{mL}$ in the experimental medium at $25^{\circ} \mathrm{C}$. The standard respiratory medium consisted of $250 \mathrm{mM}$ sucrose, $20 \mathrm{mM} \mathrm{KCl}, 5 \mathrm{mM} \mathrm{MgCl}, 5 \mathrm{mM} \mathrm{K} \mathrm{HPO}_{4}$, and $15 \mathrm{mM}$ Hepes ( $\mathrm{pH}$ 7.2). State 3 was elicited by adding $1.5 \mathrm{mM}$ adenosine $5^{\prime}$-diphosphate (ADP), and uncoupled respiration by adding $1 \mu \mathrm{M}$ carbonyl cyanide $p$ trifluoromethoxyphenyl hydrazone (FCCP); as respiratory substrates we used Malate/pyruvate, succinate, $\mathrm{NADH}$, and ascorbate/TMPD.

The effects of xenobiotics on oxidative phosphorylation were estimated by determination of ADP/O ratios. Adenosine $5^{\prime}$-triphosphate (ATP) synthesis was assayed by a HPLC procedure according to the method described by Stocchi [13].

\section{The Mitochondrial Transmembrane Potential}

A tetraphenylphosphonium $\left(\mathrm{TPP}^{+}\right)$-sensitive electrode, prepared according to Kamo et al. [14], was used to estimate the potential across the mitochondrial membrane. $\mathrm{TPP}^{+}$uptake was measured from the decreased $\mathrm{TPP}^{+}$concentration in the medium sensed by the electrode. The potential difference between the selective electrode and the reference (calomel electrode) was measured with an electrometer and recorded continuously in a Linear 1200 recorder. The voltage response of the $\mathrm{TPP}^{+}$electrode to $\log \left[\mathrm{TPP}^{+}\right]$was linear with a slope of $59 \pm 1$, at $25^{\circ} \mathrm{C}$, equating well with the Nernst equation.

Mitochondria (0.5 mg protein) were resuspended in $1 \mathrm{~mL}$ of the standard respiratory medium (the same medium as described for the oxygen consumption experiments), at $25^{\circ} \mathrm{C}$, with constant stirring. $\mathrm{TPP}^{+}$was added to a final concentration of $3 \mu \mathrm{M}$, i.e., an amount low enough to avoid any detectable toxic effects on mitochondria with the respiratory substrates. The $\mathrm{TPP}^{+}$ uptake was initiated by the addition of substrate. The $\Delta \psi$ was estimated as indicated by Kamo et al. [14] from the equation

$$
\Delta \psi(\mathrm{mV})=59 \log (v / V)-59 \log \left(10^{\Delta E / 59}-1\right)
$$

where $v$ and $V$ are the volumes of mitochondrial and incubation medium, respectively, and $\Delta E$ is the deflection of the electrode potential from the baseline. The matrix volume of $1.1 \mu \mathrm{L} / \mathrm{mg}$ mitochondrial protein was considered. The above equation was derived assuming that $\mathrm{TPP}^{+}$distribution between the mitochondria and the medium follows the Nernst equation and that the law of mass conservation is applicable. No correction was made for the passive binding of $\mathrm{TPP}^{+}$to the mitochondrial membranes, since the purpose of the experiments was to show relative changes in potentials rather than absolute values. Calibration runs in the presence of dicamba excluded any direct interference of the herbicide in the electrode signal.

\section{Enzymatic Activities}

Succinate dehydrogenase activity was measured spectrophotometrically by the reduction of 2,6dichlorophenolindophenol at $600 \mathrm{~nm}$ in the presence of phenazine methasulfate [15]. The reaction was performed in $1 \mathrm{~mL}$ of the standard reaction medium supplemented with $5 \mathrm{mM}$ succinate, $2 \mu \mathrm{M}$ rotenone, $0.1 \mu \mathrm{g}$ antimycin A, $1 \mathrm{mM} \mathrm{KCN}, 0.025 \%$ Triton X-100 at $25^{\circ} \mathrm{C}$, and $0.5 \mathrm{mg}$ protein of disrupted mitochondria (two cycles of freezing and thawing).

Succinate cytochrome $c$ reductase activity was measured spectrophotometrically [16] at $25^{\circ} \mathrm{C}$ by following 
the reduction of oxidized cytochrome $c$ by the increase in absorbance at $550 \mathrm{~nm}$. The reaction was initiated by the addition of $5 \mathrm{mM}$ succinate to $3 \mathrm{~mL}$ of the standard reaction medium supplemented with $2 \mu \mathrm{M}$ rotenone, $1 \mathrm{mM} \mathrm{KCN}, 54 \mu \mathrm{M}$ of cytochrome $c$, and $0.5 \mathrm{mg}$ protein of broken mitochondria.

Cytochrome $c$ oxidase activity was measured polarographically [17] at $25^{\circ} \mathrm{C}$ in $1 \mathrm{~mL}$ of the standard reaction medium supplemented with $5 \mathrm{mM}$ succinate, $2 \mu \mathrm{M}$ rotenone, $10 \mu \mathrm{M}$ cytochrome $c$, and $0.5 \mathrm{mg}$ protein broken mitochondria. The reaction was initiated by the addition of $5 \mathrm{mM}$ ascorbate plus $0.25 \mathrm{mM}$ TMPD.

\section{Mitochondrial Swelling}

Mitochondrial osmotic swelling [18] was monitored by detecting turbidity, at $520 \mathrm{~nm}$, on a Spectronic Genesys 2PC spectrophotometer, in a thermostatic chamber with magnetic stirring at $25^{\circ} \mathrm{C}$. Media were $44 \%$ isosmolar $(100 \%=270$ isosmolar $)$, containing $54 \mathrm{mM} \mathrm{K}$-acetate, $10 \mathrm{mM}$ Hepes, $0.1 \mathrm{mM}$ Tris-EGTA, and $0.2 \mathrm{mM}$ Tris-EDTA ( $\mathrm{pH}$ 7.1). The cuvette contained $2.0 \mathrm{~mL}$ of the reaction medium plus $0.3 \mathrm{mg}$ protein, $200 \mu \mathrm{M}$ propranolol, $10 \mu \mathrm{M}$ atractyloside, and $1 \mu \mathrm{M}$ antimycin A.

\section{Chemicals}

All reagents were of analytical grade for research. Dicamba was purchased from Riedel-Häen; 2chlorobenzoic acid and phenylacetic acid were from Merck and the other compounds were from Sigma.

\section{Treatment of the Data}

Results are presented with the values of \pm SEM from at least three independent experiments. Some figures are records of individual experiments representative of three or more replicates.

\section{RESULTS}

\section{Xenobiotic Effects on the Mitochondrial Respiration}

The effectiveness of a fixed amount of dicamba and 2-chlorobenzoic acid (Figure 1) decreased with increasing mitochondrial protein concentration when membrane potentials and rates of oxygen consumption were assayed (results not shown). For this reason the assays were always performed with a similar mitochondrial concentration.

The effects of dicamba and 2-chlorobenzoic acid on plant mitochondria respiration are displayed in
Figure 2, using succinate as the respiratory substrate; the data was obtained for state 4 and state 3 . Dicamba at $4 \mu \mathrm{mol} / \mathrm{mg}$ mitochondrial protein promotes a small stimulation on state 4 respiration, of ca. $10 \%$ of the control value, but higher concentrations significantly inhibit state 4 respiration, while 2-chlorobenzoic acid stimulates state 4 respiration as a function of concentration.

For state 3 succinate-supported respiration both compounds behave as inhibitors, dicamba being the most effective. Dicamba $(25 \mu \mathrm{mol} / \mathrm{mg}$ mitochondrial protein) promotes a decrease in state 3 of about $85 \%$, whereas 2-chlorobenzoic acid at the same concentration inhibits by $50 \%$ of the control. The observed effect of dicamba on state 4 respiration suggests that dicamba does not have any uncoupling effect.

As a control we performed the assays described in Figure 2 with a modification. First we put the mitochondria in a medium preimplemented with substrate, and 5 min later dicamba or 2-chlorobenzoic acid was added. The purpose of this control was to see if the mitochondria preincubated with dicamba prior to substrate addition provoked irreversible damage on the respiratory complexes, with a limitation in the maximal respiratory rates. From the obtained results we saw that the preincubation with dicamba before the substrate addition caused no irreversible damage on the respiratory complexes, since after 5-10 min of the dicamba addition the

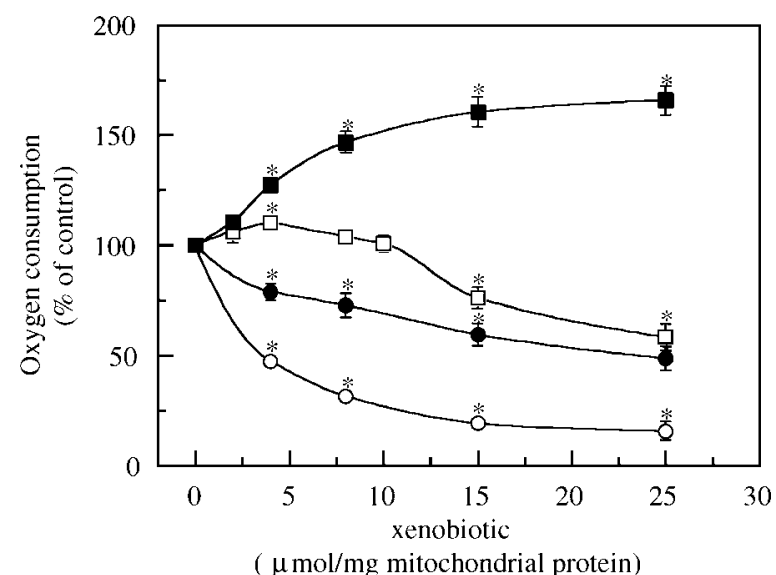

FIGURE 2. Effects of dicamba (open symbol) and 2-chlorobenzoic acid (filled symbol) on respiratory rates of mitochondria potato tuber. Mitochondria $(0.3 \mathrm{mg})$ were incubated in $1 \mathrm{~mL}$ of the respiratory standard medium, at $25^{\circ} \mathrm{C}$, in the presence of used xenobiotic concentrations $(0,2,4,8,15,25 \mu \mathrm{mol} / \mathrm{mg}$ mitochondrial protein), for $5 \mathrm{~min}$. State 4 respiration $(\square, \boldsymbol{\square})$ was initiated by the addition of $10 \mathrm{mM}$ succinate. State 3 respiration $(0, \bullet)$ energized by $10 \mathrm{mM}$ succinate was initiated by the addition of $1.5 \mathrm{mM}$ ADP. ADP was added $2 \mathrm{~min}$ after the initiation of state 4 respiration. Control values for succinatesupported respirations are expressed in $\mathrm{nmolO} \mathrm{O}_{2} /\left(\mathrm{mg}^{-1}\right.$ protein min). State 4, 49.2 ( \pm 12.2$)$; state $3,186( \pm 65.4)$. Values are the means \pm SEM of six independent experiments. *Values statistically different from control $(p<0.05)$. 
effects were observed (data not shown) and the maximal effects were identical to the results obtained when a preincubation with dicamba was performed after the substrate addition.

Oxygen consumption of controls in the absence or presence of any of the used xenobiotics was completely inhibited by the addition of antimycin A (not shown), indicating that the oxygen consumption was exclusively from the respiratory activity.

\section{Xenobiotic Effects on the Mitochondrial Transmembrane Potential}

To better evaluate the action of the tested compounds on the redox activities and phosphorylation, the alterations of the transmembrane potential $(\Delta \psi)$ developed by mitochondria at the expense of substrate oxidation were followed, as previously described (Figure 3).

After the addition of succinate, mitochondria developed a potential of about $-213 \mathrm{mV}$ and the xenobiotics promoted a $\Delta \psi$ dissipation, as a function of added concentrations.

Dicamba is the most effective agent in promoting $\Delta \psi$ dissipation, this dissipation already being apparent in the presence of $2 \mu \mathrm{mol} / \mathrm{mg}$ mitochondrial protein. Dicamba, at $25 \mathrm{mM}$, collapses $\Delta \psi$ almost completely, whereas a similar concentration of 2-chlorobenzoic acid only depresses $\Delta \psi$ by about $50 \%$ (Figures $3 \mathrm{~A}$ and $3 \mathrm{~B}$ ). Dissipation of $\Delta \psi$ is not a consequence of a progressive stimulation of state 4 respiration, as could be expected if $\Delta \psi$ collapse were only the result of a membrane permeabilization to protons.

(A)

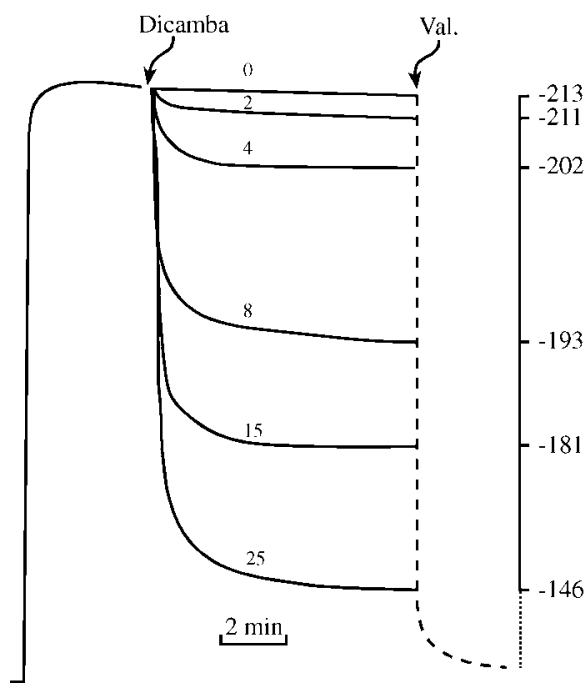

\section{Xenobiotic Effects on the Oxidation of Different Substrates}

The oxidation of different substrates for FCCPstimulated respiration in the presence of dicamba and 2-chlorobenzoic acid ( $15 \mu \mathrm{mol} / \mathrm{mg}$ mitochondrial protein) were inhibited for most of the used substrates, the exception being ascorbate/TMPD where no significant inhibition was observed (Figure 4).

In mitochondria respiring malate/pyruvate (Figure 4), dicamba at $15 \mu \mathrm{mol} / \mathrm{mg}$ mitochondrial protein depresses the redox chain activity by about $54 \%$, whereas 2-chlorobenzoic acid decreases the activity by $37 \%$. With succinate (Figure 4), the inhibition observed for dicamba and for 2-chlorobenzoic acid at the maximum concentration used $(15 \mu \mathrm{mol} / \mathrm{mg}$ mitochondrial protein) were 65 and $44 \%$ respectively. When NADH or ascorbate/TMPD was used as substrate (Figure 4), no significant differences were observed between dicamba and 2-chlorobenzoic acid. Furthermore, respiration supported by exogenous NADH was inhibited by about $30 \%$, but no significant inhibition was observed for respiration promoted by ascorbate/TMPD, which is linked to cytochrome $c$ oxidase.

\section{Xenobiotic Effects on the Oxidative Phosphorylation}

The influence of dicamba and 2-chlorobenzoic acid on phosphorylation was tested using succinate as the respiratory substrate (Figure 5). In the absence of any xenobiotic we obtained an ADP/O of about 1.8, but when dicamba or 2-chlorobenzoic acid was added at
(B)

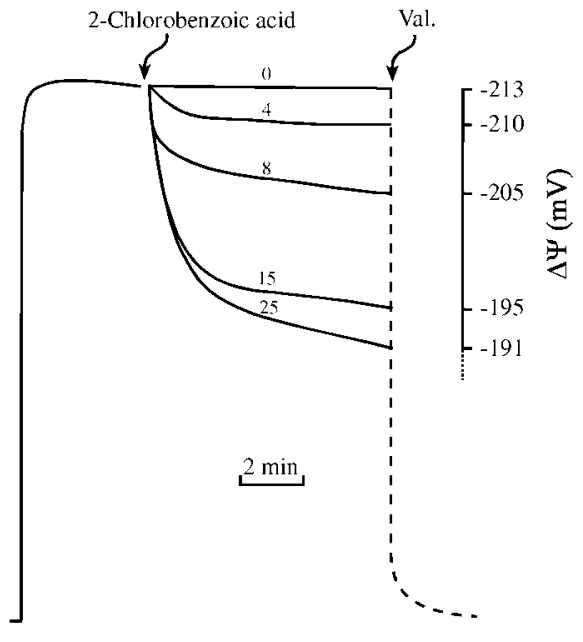

FIGURE 3. Effects of dicamba (A) and 2-chlorobenzoic acid (B) on transmembrane potential $(\Delta \psi)$ supported by succinate. Mitochondria (0.3 $\mathrm{mg}$ ) were added to the standard respiratory medium supplemented with $3 \mu \mathrm{M} \mathrm{TPP}{ }^{+}$. Xenobiotics were added at the indicated concentrations $(0,2,4,8,15,25 \mu \mathrm{mol} / \mathrm{mg}$ mitochondrial protein). Valinomycin was added at the end of each assay to elicit complete collapse of membrane potential. The traces represent typical recordings from several experiments with different mitochondrial preparations. 


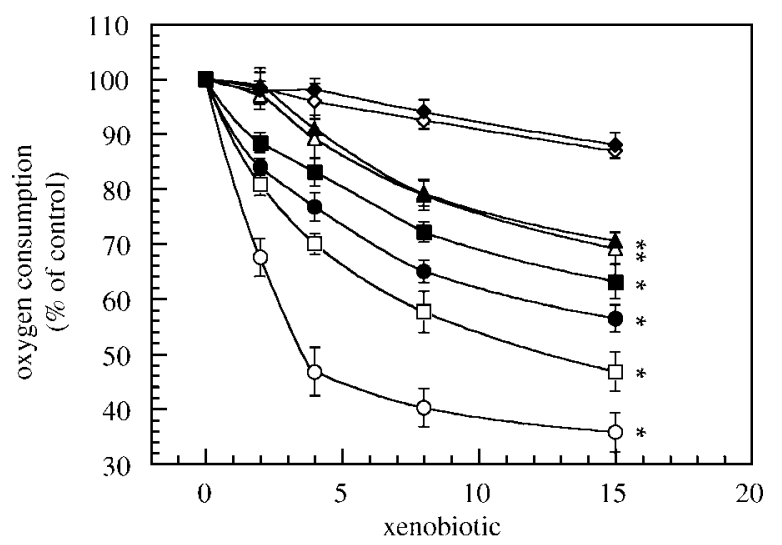

( $\mu \mathrm{mol} / \mathrm{mg}$ mitochondrial protein)

FIGURE 4. Effects of dicamba (open symbol) and 2-chlorobenzoic acid (filled symbol) on FCCP-uncoupled respiration for malate $(\square, \boldsymbol{\square})$, succinate $(\mathrm{O}, \boldsymbol{\square})$, exogenous NADH $(\Delta, \boldsymbol{\Delta})$, and ascorbate/TMPDsupported respiration $(\diamond, \diamond)$ in potato tuber mitochondria. Assay media: malate (20 mM malate, $4 \mathrm{mM}$ pyruvate, $1 \mathrm{mM}$ TPP, and 1 mM NAD ${ }^{+}$); succinate (10 $\mathrm{mM}$ succinate); exogenous NADH (1 mM $\mathrm{NADH})$; ascorbate/TMPD (10 $\mathrm{mM}$ ascorbate and $0.75 \mathrm{mM}$ TMPD). Control values are expressed in $\mathrm{nmol}_{2} /(\mathrm{mg}$ protein min): malate, 120 ( \pm 11.0$)$; succinate, $198( \pm 30.1)$; exogenous NADH, $158( \pm 24.3)$; ascorbate/TMPD, $298( \pm 38.0)$. Values are the means \pm SEM of three independent experiments.

$2 \mu \mathrm{mol} / \mathrm{mg}$ mitochondrial protein the ADP/O decreased to 0.8 and to 1.2 , respectively. For $15 \mu \mathrm{mol} / \mathrm{mg}$ mitochondrial protein the inhibition observed on the ATP synthesis were 74 and $40 \%$, respectively for dicamba and for 2-chlorobenzoic acid. The sharp depression induced by 2-chlorobenzoic acid on the $\mathrm{ADP} / \mathrm{O}$ was not accompanied by a sharp depression

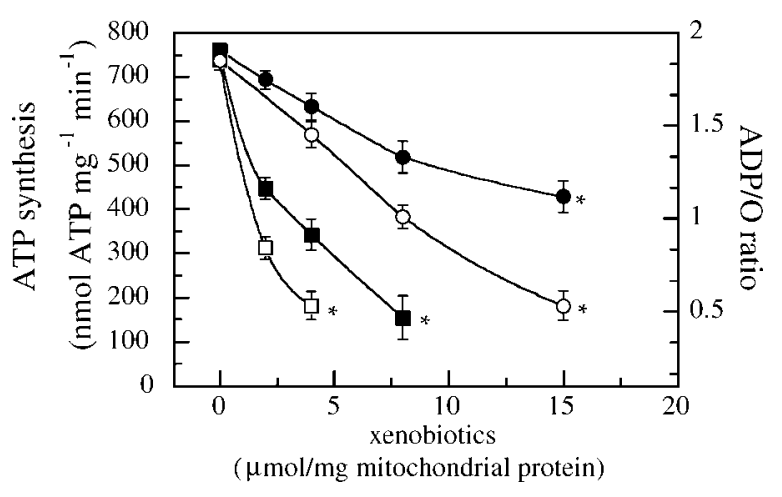

FIGURE 5. Effects of dicamba (open symbol) and 2-chlorobenzoic acid (filled symbol) on the oxidative phosphorylation of potato tuber mitochondria supported by succinate. Synthesis of $\operatorname{ATP}(\bullet, 0)$, $\operatorname{ADP} / \mathrm{O}$ ratio $(\boldsymbol{\square}, \square)$. Mitochondria $(1 \mathrm{mg})$ were incubated in $2 \mathrm{~mL}$ reaction mediums (described in the Methods Section), at $25^{\circ} \mathrm{C}$. Control values for ATP synthesis $760( \pm 82.0) \mathrm{nmol} \mathrm{ATP} /(\mathrm{mg}$ mitochondrial protein $\mathrm{min}) ; \mathrm{ADP} / \mathrm{O} 1.85( \pm 0.053)$. Assays were performed in the conditions described in the Methods Section. Values are the means \pm SEM of three independent experiments. *Values statistically different from control $(p<0.05)$. of ATP synthesis, since more than $50 \%$ synthesis was still observed at $15 \mu \mathrm{mol} / \mathrm{mg}$ mitochondrial protein, while ADP/O was nonmeasurable at this concentration. ADP/O was already nonmeasurable for dicamba at $8 \mu \mathrm{mol} / \mathrm{mg}$ mitochondrial protein, but ATP synthesis was still measurable at $15 \mu \mathrm{mol} / \mathrm{mg}$ mitochondrial protein.

\section{Xenobiotic Effects on the Respiratory Complexes (II, III and IV) Activity}

Studies of enzymatic activities of respiratory complexes II, III, and IV localized the components of the mitochondrial respiratory chain affected by dicamba and 2-chlorobenzoic acid (Figure 6). The effect of xenobiotics on the redox activities helps us to understand and explain not only the interaction with redox complexes but also the changes in the membrane potential. Dicamba (25 $\mu \mathrm{mol} / \mathrm{mg}$ mitochondrial protein) markedly inhibits the succinate cytochrome $c$ reductase activity by about $50 \%$ as well as succinate dehydrogenase activity by about $35 \%$. The inhibition induced by 2-chlorobenzoic acid, at the same concentration, was only about 30 and $20 \%$, respectively, for succinate cytochrome $c$ reductase and succinate dehydrogenase. The terminal segment of the respiratory chain, cytochrome $c$ oxidase, was not significantly inhibited by either xenobiotic.

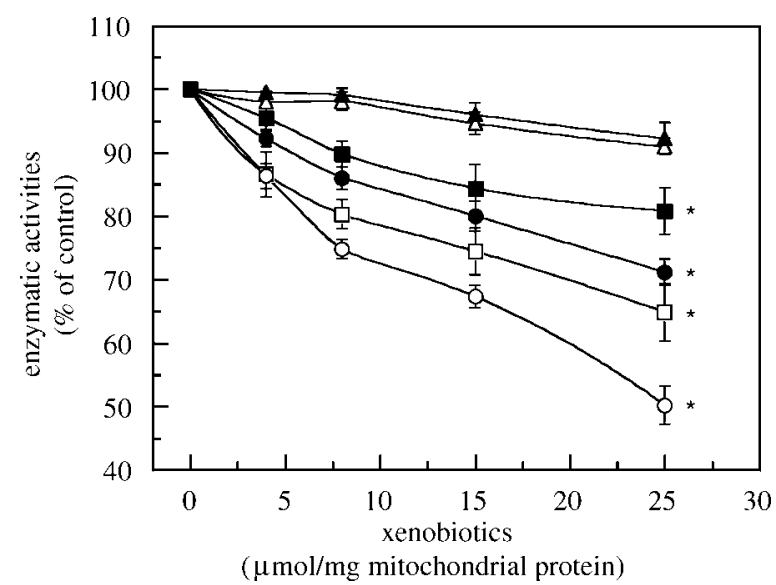

FIGURE 6. Effects of dicamba (open symbol) and 2-chlorobenzoic acid (filled symbol) on the respiratory complexes: succinate dehydrogenase $(\boldsymbol{\square}, \square)$, succinate cytochrome $c$ reductase $(\bullet, 0)$, cytochrome $c$ oxidase $(\boldsymbol{\Lambda}, \Delta)$. Mitochondria $(1 \mathrm{mg})$ were incubated in $2 \mathrm{~mL}$ reaction mediums (described in the Methods Section), at $25^{\circ} \mathrm{C}$. Assays were performed in the conditions described in the Methods Section. Control values: succinate dehydrogenase, $230( \pm 12.2) \mathrm{nmol} \mathrm{O}_{2} /(\mathrm{mg}$ mitochondrial protein $\mathrm{min}$ ); succinate cytochrome $c$ reductase, 1.8 $( \pm 0.042)$ nmol cytochrome $c$ reduced/(mg mitochondrial protein $\mathrm{min})$; cytochrome $c$ oxidase, $435( \pm 42.3) \mathrm{nmol} \mathrm{O}_{2} /(\mathrm{mg}$ mitochondrial protein $\mathrm{min}$ ). Values are the means \pm SEM of three independent experiments. 


\section{Proton-Dependent Mitochondrial Swelling}

Mitochondrial swelling was followed in potassium acetate $(54 \mathrm{mM})$ isosmolar medium in the presence of different xenobiotics (Figure 7). The maximal valinomycin-dependent swelling stimulation was observed upon addition of FCCP $(1 \mu \mathrm{M})$. Mitochondrial swelling occurs if a protonophore is present to promote the passage of protons from the matrix to the extramitochondrial reaction medium, permitting further acetate and $\mathrm{K}^{+}$influx [19]. Therefore, swelling promoted by valinomycin under the action of added xenobiotics is a consequence of protonophoric actions.

Both the tested xenobiotics can increase the permeability of inner mitochondria membrane to protons. Dicamba (Figure 7A) was the most efficient in inducing swelling as a function of concentration; 2-chlorobenzoic acid (Figure 7B) also induces swelling but in a smaller degree when compared with dicamba.

\section{DISCUSSION}

Mitochondrial transmembrane potential studies are of major importance in relating the effect of xenobiotics to the mitochondrial oxidative phosphorylation, since $\Delta \psi$ represents the main component of the electrochemical gradient and accounts for more than $90 \%$ of the total available energy [19] on nonphotosynthetic tissues.

From the strong $\Delta \psi$ dissipation promoted by the dicamba (Figure $3 \mathrm{~A}$ ) a strong stimulation should be expected on the state 4 respiration, since with the $\Delta \psi$ dissipation the imposed restriction by the proton motive force can be raised, but the obtained result on the state $4 \mathrm{O}_{2}$ consumption did not agree with a strong $\Delta \psi$ dissipation promoted by an increase on the proton leak. Even for 2-chlorobenzoic acid the stimulation observed in the state 4 respiratory rate is much lower than could be expected from the observed $\Delta \psi$ dissipation [20]. Therefore, we believe that the greater part of the $\Delta \psi$ dissipation must be as a result of an inhibitory effect on the redox chain complexes.

The results obtained on the state 3 (Figure 2) and on the uncoupled respiration (Figure 4) clearly indicate an inhibitory effect on some of the respiratory complexes.

The respiration rate was differentially inhibited by the two xenobiotics, depending on the nature of the respiratory substrate used. The exception was observed with the mitochondria respiring ascorbate/TMPD and exogenous $\mathrm{NADH}$, since no significant difference was observed between dicamba and 2-chlorobenzoic acid (Figure 4). The results in Figure 4 points to the conclusion that complexes I, II, and III can be strongly inhibited, while the terminal segment of the respiratory chain (cytochrome $c$ oxidase) cannot be significantly affected by these two xenobiotics. These results are further supported by the results obtained for succinate dehydrogenase, succinate cytochrome $c$ reductase, and cytochrome $c$ oxidase (Figure 6). Inhibition of succinate-supported respiration by dicamba and 2chlorobenzoic acid $(15 \mu \mathrm{mol} / \mathrm{mg}$ mitochondrial protein) was 55 and $35 \%$, respectively, while at this concentration the inhibition of the respiration supported by exogenous NADH was about $30 \%$ for both xenobiotics. Considering that the oxidation of both substrates, succinate, pyruvate/malate, and exogenous NADH have a common electron-transfer pathway, it is tempting to conclude that the two xenobiotics, especially dicamba, may act on substrate transport [21], since the oxidation of the exogenous NADH was less inhibited than the oxidation of succinate or malate, and only NADH does not need to cross the inner mitochondrial membrane to be oxidized. Furthermore, the difference in the inhibition (\%) observed between Figure 4 (oxygen consumption on FCCP-uncoupled respiration) and Figure 6
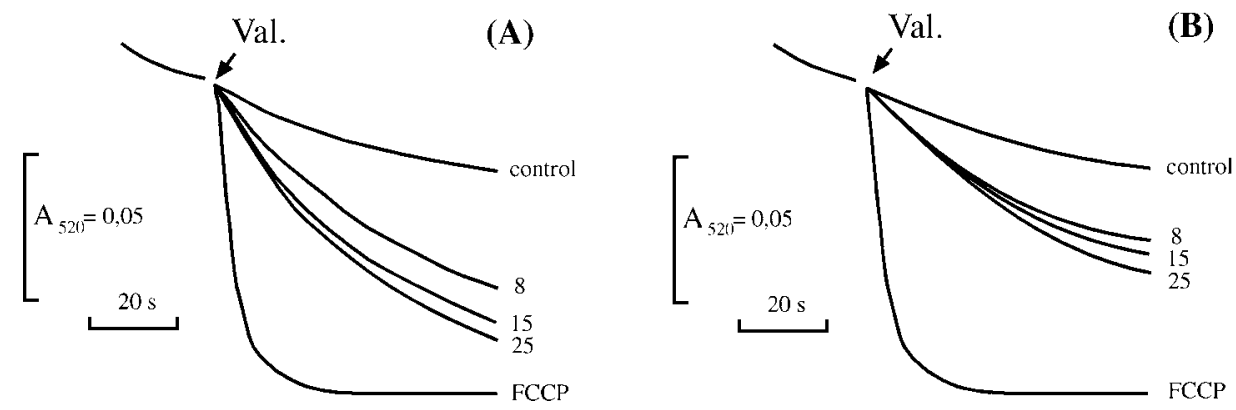

FIGURE 7. Effects of dicamba (A) and 2-chlorobenzoic acid (B) on the mitochondrial swelling. Swelling in $44 \%$ isosmolar potassium acetate ( $\mathrm{pH}$ 7.1) supplemented with antimycin $(1 \mu \mathrm{M})$, atractyloside $(10 \mu \mathrm{M})$ and propranolol $(200 \mu \mathrm{M})$, with BSA (0.1\%). Xenobiotics were added as indicated in the figure $(8,15,25 \mu \mathrm{mol} / \mathrm{mg}$ mitochondrial protein). Control represents the assay without any xenobiotic or FCCP. The line indicated by FCCP represents an experiment in which $1 \mu \mathrm{M}$ FCCP was added; valinomycin $(1 \mu \mathrm{M})$ was added in all the experiments like it is indicated. The traces are representative of a group of at least three independent experiments. 
(respiratory complexes activities) is certainly the result of an inhibitory effect on the substrate transport across the membrane, since the enzymatic activities are obtained with disrupted mitochondria and for this reason membrane transport is not necessary, whereas FCCP-uncoupled respiration was obtained with intact mitochondria. We did not evaluate the activity of the exogenous NADH dehydrogenase, since in plant mitochondria three different NADH dehydrogenases are present [22].

Both xenobiotics can stimulate a proton leak through the mitochondrial inner membrane as was demonstrated by the swelling assays (Figure 7).

Independent of actions on the different complexes of the redox chain, we can also see from Figure 5 that dicamba and 2-chlorobenzoic acid can both interact with the phosphorylation system, by a similar mechanism to that already demonstrated for other xenobiotics $[23,24]$. The effect on the phosphorylation system is probably due to two different mechanisms; first, a direct effect on the $\mathrm{F}_{0}-\mathrm{F}_{1}$ ATPase, since ATPase activity was also inhibited (data not show), and second, by an inhibition on the nucleotides carrier and inorganic phosphate carrier, since the inhibition observed on the ATP synthase activity (Figure 5) was much greater than that obtained for ATPase.

In our laboratory we have tested the effect of a number of xenobiotics, including dicamba, on cell growth of nongreen potato tuber callus, and the results show that dicamba did not stimulate superoxide dismutase, catalase, or glutathione reductase (data not shown). Therefore, on the basis of these results we can assume that dicamba and 2-chlorobenzoic acid metabolisms do not produce any significant reactive oxygen species. Moreover, oxygen consumption supported by succinate of controls, in the absence or presence of any of the used xenobiotics, was completely inhibited by the addition of antimycin A (not shown), indicating that the oxygen consumption came exclusively from the respiratory activity.

If we compare the sensitivity of mitochondria to dicamba or to 2-chlorobenzoic acid with the sensitivity obtained with other compounds, like bromosulfophtalein [24], dinoseb [25], styrylchromone [26], and with many others, we can see that mitochondrial bioenergetics are much less affected by dicamba and 2-chlorobenzoic acid (a difference in the order of $10^{3}$ ). This difference in the sensitivity is due to the lower lipophilicity of dicamba and 2-chlorobenzoic acid, and therefore, their incorporation in membrane lipid domains is much poorer than other xenobiotics. At higher concentrations like those used in our experiments, some dicamba can certainly incorporate into the membrane, the aromatic portion being immersed into the hydrophobic part, whereas the polar portion of the molecule remains in the hydrophilic surface. In fact, we tried to measure the partition of dicamba and 2chlorobenzoic acid into submitochondrial particles, using a spectroscopic method described by Videira et al. [27], but it was impossible to measure because the level of incorporation was very small.

The differences observed in the effects of dicamba and 2-chlorobenzoic acid should be related to the differences in the chemical structure itself, which result in different lipophilicity. As a consequence those xenobiotics should present different extensions of mitochondrial membrane incorporation. Therefore, these differences should result from a different extension of a direct interaction with functional membrane proteins and/or boundary lipids, as it has been known for a long time that the functionality of many membranes proteins are strongly associated with the surrounding lipids [28].

In conclusion, the inhibitory action of dicamba and 2-chlorobenzoic acid on the phosphorylation efficiency of mitochondria could be, in the last analysis, due to an incorporation of dicamba into the mitochondrial membranes, causing an alteration on the surface charge density and a disturbance in the physicochemical and structural properties of the inner membrane, resulting in an unequivocal disturbance in the electron delivery between redox complexes (in particular between II and III) in the phosphorilative system, and, in addition, an increase of the permeability to the protons.

\section{REFERENCES}

1. Blondin GA, Knobelock LM, Read HW, Harkin JM. Mammalian mitochondria as in vitro monitors of water quality. Bull Environ Contam Toxicol 1987;38:467-474.

2. Knobeloch LM, Blondin GA, Harkin M. Use of submitochondrial particles for prediction of chemical toxicity in man. Bull Environ Contamination Toxicol 1990;44:661668.

3. Argese E, Betticol C, Ghelli A, Todescheni R, Miana P. Submitochondrial particles as toxicity biosensors of chlorophenols. Environ Toxicol Chem 1995;14:363-368.

4. Neuburger M, Journet E-P, Bligny R, Carde J-P, Douce R. Purification of plant mitochondria by isopycnic centrifugation in density gradients of percoll. Arch Biochem Biophys 1982;217:312-323.

5. Moreland DE. Mechanisms of action of herbicides. Ann Rev Plant Physiol 1980;31:597-638.

6. Moreland DE. Weed Physiol. Boca Raton, FL: CRC Press; 1987.

7. Wright JP. Use of membrane potential measurements to study mode action of diclofop-methyl. Weed Sci 1994;42:285-292.

8. Wall DA. Potato (Solanum-Tuberosum) response to simulated drift of dicamba, clopyralid and tribenuron. Weed Sci 1994;42:110-114.

9. Espandiari P, Thomas VA, Glauert HP, Obrien M, Noonan D, Robertson LW. The herbicide dicamba (2-methoxy-3,6dichlorobenzoic acid) is a peroxisome proliferator in rats. Fundam App Toxicol 1995;26:85-90. 
10. Fairchild JF, Ruesseler DS, Haverland PS, Carlson AR. Comparative sensitivity of Selenastrum capricornutum and Lemna minor to sixteen herbicides. Arch Environ Contam Toxicol 1997;32:353-357.

11. Gornall AG, Bardawill CJ, David MM. Determination of serum proteins by means of the biuret. J Biol Chem 1949;177:751-766.

12. Estabrook RW. In: Racker E, editor. Membranes of Mitochondria and Chloroplasts. New York: Van Nostrand; 1967. pp 172-212.

13. Stocchi V, Cucchiarini L, Magnami M, Chiarantini L, Palma P, Crescentini G. Simultaneous extraction and reverse-phase high-performance liquid chromatographic determination of adenine and pyridine nucleotides in human red blood cells. Anal Biochem 1985;146:118-124.

14. Kamo N, Muratsugu M, Hongoh R, Kobatake V. Membrane potential of mitochondria measured with an electrode sensitive to tetraphenylphosphonium and relationship between proton electrochemical potential and phosphorylation potential in steady state. J Membrane Biol 1979;49:105-121.

15. Singer TP. Determination of the activity of succinate, $\mathrm{NADH}$, choline and glycerophosphate dehydrogenases. Met Biochem Anal 1974;22:123-175.

16. Tisdale HD. Preparation and Properties of succiniccytochrome $c$ reductase (complex II and III). Methods Enzymol 1967;10:213-215.

17. Brautigan DL, Ferguson-Miller S, Margoliash E. Mitochondrial cytochrome $c$ : Preparation and activity of native and chemically modified cytochrome $c$. Methods Enzymol 1978;53:128-164.

18. Beavis AD, Vercesi AE. Anion uniport in plant mitochondria is mediated by a $\mathrm{Mg}^{2+}$-insensitive inner membrane anion channel. J Biol Chem 1992;267:3097-3087.
19. Nicholls DG. Bioenergetics. An Introduction to the Chemiosmotic Theory. London: Academic Press; 1982.

20. Lambert CE, Bondy SC. Effects of MPTP, $\mathrm{MPP}^{+}$and paraquat on mitochondrial potential and oxidative stress. Life Sci 1989;44:1277-1284.

21. LaNoue KF, Schoolwerth AC. Metabolite transport in mitochondria. Annu Rev Biochem 1979;48:871-922.

22. Møller IM. Membrane-bound NAD(P)H dehydrogenases in higher plant cells. Ann Rev Plant Physiol 1987;37:309334.

23. Burr R, Schwenk M, Pfaff E. Interaction of bromosulfophthalein with mitochondrial membranes-inhibition of respiration. Biochem Pharmacol 1977;26:461-466.

24. Schwenk M, Bur R, Baur H, Pfaff E. Interaction of bromosulfophthalein with mitochondrial membranes: Effect on ion movements. Biochem Pharmacol 1977;26:825832.

25. Palmeira CM, Moreno AJ, Madeira VMC. Interactions of herbicides dinoseb and 2,4-D with liver mitochondrial bioenergetics. Toxicol Appl Pharmacol 1994;127:5057.

26. Peixoto F, Barros NA, Silva AMS. Interactions of a new 2-styrylchromone with mitochondrial oxidative phosphorylation. J Biochem Mol Toxicol 2002;16,220226.

27. Videira RA, Antunes-Madeira MC, Custódio JBA, Madeira VMC. Partition of DDE in synthetic and native membranes determined by ultraviolet derivative spectroscopy. Biochem Biophys Acta 1995;1238:2228.

28. Antunes MMC, Madeira VMC. Membrane fluidity as affected by the organochloride insecticide DDT. Biochim Biophys Acta 1990;1023:469-474. 